\title{
INFLUÊNCIA DE DIFERENTES NÍVEIS DE CÁLCIO EM PLANTAS DE ESTILOSANTES (Stylosanthes guyanensis (Aubl.) Sw. CV. COOK), AVALIADOS ATRAVÉS DE ALGUNS PARÂMETROS FISIOLÓGICOS
}

\author{
J.D. RODRIGUES; S.D. RODRIGUES; M.E.A. DELACIIIAVE; J.F. PEDRAS; C.S.F. BOARO \\ Dep. de Botânica - Instituto de Biociências/UNESP - CEP: 18618-000-Botucatu,SP. \\ E.O. ONO \\ Biologa, M.Sc., Pos-Graduando do Curso de Ciências Biologicas - IB/UNESP - CEP: 18618-000-Botucatu,SP.
}

\begin{abstract}
RESUMO: O objetivo do presente trabalho foi estudar os efeitos de diferentes níveis de cálcio, no desenvolvimento de plantas de Stylosanthes guyanensis (Aubl.) Sw. cv. Cook, através de alguns parâmetros que compõem a análise fisiologica de crescimento. Para tal foram empregados 4 tratamentos a saber: T1 (200 mg de cálcio/litro); T2 (133,33 mg de cálcio/litro); T3 (66,66 mg de cálcio/litro) e T4 (omisso em cálcio). O trabalho foi montado em cultivo hidropônico, utilizando-se a solução nutritiva $n^{\circ} 1$ de Hoagland \& Arnon e conduzido em casa de vegetação. Foram realizadas observações em intervalos entre 5 coletas, distribuidas da seguinte maneira: intervalo I (entre $24^{\circ}$ e $38^{\circ}$ dia pós-germinação; intervalo II ( do $38^{\circ}$ ao $52^{\circ}$ dia); intervalo III (do $52^{\circ}$ ao $66^{\circ}$ dia) e intervalo IV (do $66^{\circ}$ ao $80^{\circ}$ dia). Os parâmetros estudados foram: a. taxa assimilatória líquida (TAL); b. taxa de crescimento relativo (TCR) e c. razāo alfa. Dos resultados obtidos pode-se concluir que soluções com 200 e 133,33 mg de cálcio/litro, foram mais eficientes em promover o desenvolvimento de plantas de estilosantes.

Descritores: estilosantes, análise de crescimento, cálcio, nutrição mineral.
\end{abstract}

\section{INFLUENCE OF DIFFERENT CAICIUM LEVEIS IN FSTILO PI.ANTS (Stylosanthes guranensis (Aubl.) Sw. CV. COOK), EVAIJATED THROUGII SOME PIIYSIOLOGICAL PARAMETERS}

\begin{abstract}
This paper reports a greenhouse study on the influence of different levels of calcium on the development of Stylosanthes guyanensis (Aubl.) Sw. cv. Cook plants, through physiological parameters related to plant growth. Four treatments were used: T1 (200 mg of calcium per litre); T2 (133.33 mg of calcium per litre); T3 (66.66 $\mathrm{mg}$ of calcium per litre) and $\mathrm{T} 4$ (without calcium). The experiment was carried out in hydroponic culture whith IIoagland \& Amon $n^{\circ} 1$ solution. Observations were made in five intervals: interval I (between the 24th. and 38th. days after germination); interval II (between the 38th. and 52th. days after germination); interval III (between the 52th. and 66th. days after germination); and interval IV (between the 66th. and 80th. days after germination). The parameters studied were: a. net assimilation rate (NAR); b. relative growth rate (RGR); and c. alfa rate (alfa $R)$. It could be concluded that solutions with 200 and $133.33 \mathrm{mg}$ of calcium per litre were more effective to improve the development of estilo plants.
\end{abstract}

Key Words: estilo plants, growth analysis, calcium, mineral nutrition.

\section{INTRODUÇño}

Apesar do rebanho bovino brasileiro ser, numericamente, um dos maiores do mundo, o desenvolvimento pecuário encontra-se defasado, devido à pequena produlividade. Esta constatação tem sua origem em vários fatores, dentre os quais destaca-se a má qualidade das pastagens, associada à baixa fertilidade dos solos (BARRIGA, 1979).

VASCONCELOS et al. (1974), afirmaram que no Brasil, a exploração pecuária apóia-se quase que exclusivamente na atualização de pastagens, como recurso para a nutrição animal.

Sci. agric., Piracicaba, 50(1):45-57, fev./majo, 1993
A introdução das leguminosas em pastagens de gramíneas, propicia melhora quantitativa na produção de forragens, aumentando a capacidade de suporte do pasto. Para BARRIGA (1979), as leguminosas forrageiras são a fonte mais econômica de proteínas para os rebanhos, além de contribuírem para o suprimento de nitrogênio ao sistema solo-planta.

Face ao exposto, percebe-se a importância do Stylosanthes gtyanesis (Aubl.) Sw. cv. Cook, como forrageira, entre as leguminosas tropicais, a qual é a mais encontrada e utilizada no Brasil, como uma alternativa para o aumento da produtividade de pastagens tropicais (WHYTE et al., 1968). 
No entanto, aparecem na literatura contradições a respeito das exigências nutricionais dessa leguminosa, como a calagem que, segundo ANDREW (1962), se faz necessária para um aumento da produção de estilosantes. EIRA et al.(1970) verificaram ainda que o cálcio, acrescido de magnésio, aumentou o nitrogênio percentual de Macroptilium atroporpureum e Glycine javanica. Além disso, FRANÇA \& CARVALHO (1970), trabalhando com 5 leguminosas de clima tropical, verificaram que a ausência de calagem determinou em todas as leguminosas, uma diminuição da fixação de nitrogênio e da matéria seca. Ao contrário, NORRIS (1959) relatou que as leguminosas forrageiras tropicais adaptam-se em solos ácidos, possuindo capacidade elevada de extração de nutrientes.

A análise de crescimento foi definida por MAGALHÃES (1979) como o método que descreve as condições morfofisiológicas da planta, em diferentes intervalos de tempo, entre duas amostragens sucessivas e se propõe a acompanhar a dinâmica da produção fotossintética, avaliada através do acúmulo de matéria seca.

Frente ao exposto acima, considerando-se a importância das leguminosas no enriquecimento protéico de pastagens e o baixo nível de cálcio nos solos onde estas são cultivadas, propõe-se o estudo do comportamento fisiológico do Stylosanthes givyanensis (Aubl.) Sw. cv. Cook, submetido a condições de diferentes níveis de cálcio, avaliado através de alguns parâmetros fisiológicos que compõem a análise de crescimento.

\section{MATERIAL E MÉTODOS}

Inicialmente, as sementes de Stylosanthes guyanensis (Aubl.) Sw. cv. Cook foram colocadas em bandejas de plástico, contendo algodão e papel de filtro umedecidos com água desmineralizada $\mathrm{e}$ colocadas em câmara climática à uma temperatura de $20^{\circ} \mathrm{C}$, até a emergência da radicula, aproximadamente $5 \mathrm{~mm}$ de comprimento em média.

Posteriormente, as sementes germinadas foram transferidas para bandejas, contendo vermiculita como substrato e irrigadas com solução nutritiva $n^{\circ} 1$ de HOAGLAND \& ARNON (1950), diluída a 1/5, objetivando evitar possíveis transtornos fisiológicos às plântulas.

Com a idade de 10 dias, as plantas foram colocadas em vasos de plástico, em cultivo hidropônico, sob arejamento constante, contendo solução nutritiva $n^{\circ} 1$ de HOAGLAND \& ARNON (1950), completa e com diferentes níveis de cálcio. A partir dessa solução nutritiva, contendo $200 \mathrm{mg}$ de cálcio/litro (completa), foram preparadas mais duas soluções com $2 / 3$ e 1/3 da quantidade de cálcio da inicial e uma outra solução omissa em cálcio, de onde resultaram os seguintes tratamentos:

T1 - solução nutritiva com $200 \mathrm{mg}$ de cálcio/litro T2 - solução nutritiva com 133,33 mg de cálcio/litro T3 - solução nutritiva com $66,66 \mathrm{mg}$ de cálcio/litro T4 - solução nutritiva omissa em cálcio

O efeito dos níveis de cálcio foi verificado através dos seguintes parâmetros físiológicos da análise de crescimento:

a. taxa assimilatória líquida (TAL), expressa em $\mathrm{g} / \mathrm{cm}^{2}$.dia e que reflete a influência do sistema assimilador envolvido na produção de matéria seca, estimando a fotossíntese líquida (BOARO, 1986), definida pela relação:

$T_{n \rightarrow n-1} \frac{\left[\mathrm{PST}_{n}-\mathrm{PST}_{n-1}\right] \cdot\left[\ln A F_{n}-\ln A F_{n-1}\right]}{\left[A F_{n}-A F_{n-1}\right] \cdot\left[t_{n}-t_{n-1}\right]}$

onde:

$\mathrm{n}=$ número da coleta

$\mathrm{t}=$ tempo, entre coletas, em dias

PST = matéria seca total, em gramas

$\mathrm{AF}=$ área foliar, $\mathrm{em} \mathrm{cm}^{2}$

b. taxa de crescimento relativo (TCR), expressa em g/g.dia, refletindo o aumento da matéria orgânica seca em gramas, de uma planta ou de qualquer orgão desta, num intervalo de tempo. É funcão do tamanho inicial, ou seja, de material pré existente (BENINCASA, 1988; RODRIGUES, 1990), sendo definida pela expressão:

$$
\begin{aligned}
& \mathrm{RAF}_{\mathrm{n}}+\mathrm{RAF}_{\mathrm{n}-1} \\
& T C R=T A L \quad x \\
& n \rightarrow n-1 \quad 2
\end{aligned}
$$

onde:

$\mathrm{n}=$ número da coleta

TAL $=$ taxa assimilatória líquida

$\mathrm{RAF}=$ razão de área foliar 
c. Razão alfa, expressa $\mathrm{em} \mathrm{cm}^{2} / \mathrm{g}$, que indica a direção dos assimilados (RODRIGUES, 1987; RODRIGUES, 1990), definida pela expressão:

$$
\underset{n \rightarrow n-1}{\operatorname{Razão~alfa}}=\frac{\operatorname{lnAF}_{n}-\mathbb{I N ~ A F}_{n-1}}{\operatorname{lnPST}_{n}-\operatorname{hPST}_{n-1}}
$$

onde:

$\mathrm{n}=$ número de coleta

$\mathrm{AF}=$ Área foliar, em $\mathrm{cm}^{2}$

PST = peso da matéria seca total, em $\mathrm{g}$

Para o cálculo destes três parâmetros fisiológicos da análise de crescimento, utilizaram-se os dados de área foliar, matéria seca e razão de área foliar (RAF):

a. área foliar (AF): imprimindo-se os contomos das folhas de papel heliográfico, de acordo com SESTAK et al. (1971) e RODRIGUES (1990). Em cada sub-parcela, a área foliar foi definida como o somatório de todas as folhas das duas plantas.

b. matéria seca (PST): determinada através de secamento do material vegetal, em estufa com circulação forçada de ar, a $60-70^{\circ} \mathrm{C}$, até peso constante (BENICANSA, 1988), medido em cada órgão, individualmente: raiz, caule e folhas. Em cada sub-parcela, a matéria seca foi definida como a soma da matéria seca das duas plantas do vaso.

c. razão de área foliar (RAF): definida como o quociente entre a área foliar (AF) e matéria seca da planta (PST), expressa $\mathrm{em} \mathrm{cm}^{2} / \mathrm{g}$ (RODRIGUES, 1982).

Essas observações foram realizadas nos intervalos entre as 5 coletas, efetuadas a cada 14 dias, distribuídos da seguinte maneira: intervalo I (entre o $24^{\circ}$ ao $38^{\circ}$ dia pós-germinação); intervalo II $\left(38^{\circ}\right.$ a $\left.52^{\circ} \mathrm{dia}\right)$; intervalo III $\left(52^{\circ}\right.$ ao $\left.66^{\circ} \mathrm{dia}\right) \mathrm{e}$ intervalo de IV $\left(66^{\circ}\right.$ ao $\left.80^{\circ} \mathrm{dia}\right)$.

$O$ experimento, inteiramente casualizado, obedeceu ao delineamento de parcelas sub-divididas, considerando-se as cinco coletas como parcelas, sendo os tratamentos definidos como sub-parcelas. Não foram realizadas as análises de variância, uma vez que não se pode afirmar que essas variáveis, por serem calculadas, obedeçam às pressuposições básicas para a realização dessa análise (BANZATTO \& KRONKA, 1989).

\section{RESULTADOS E DISCUSSÃO}

\section{Taxa Assimilatoria Ĺ́quida}

Os resultados obtidos para a taxa assimilatória líquida (TAL), nos quatro tratamentos, encontram-se na TABELA 1 e Figura 1. A análise dessa figura mostra diminuição da TAL com a progressão do ciclo de desenvolvimento do estilosantes, estando essa diminuição concentrada entre o primeiro e o segundo intervalo, sendo menor entre o segundo e os demais intervalos, que foram considerados para o cálculo da TAL.

Esses resultados conflitam com alguns relatados na literatura, como os de ASCENIO \& FARGAS (1973), que, em feijão, encontraram aumentos na TAL até a idade de 62 dias, enquanto BOARO (1986), trabalhando com a mesma espécie, não encontrou variações nos valores de TAL, durante o desenvolvimento dessa planta. Para KUMURA \& NANIWA (1965), a TAL aumenta com o crescimento das folhas, atingindo um valor máximo, diminuindo ao atingir a senescência. No entanto, outros autores apresentaram resultados concordantes com os aqui obtidos, como WATSON (1952), KUMURA \& NANIWA (1965), BUTTERY (1964), os quais obtiveram diminuição na taxa assimilatória líquida, com a progressão do ciclo do vegetal. Em soja, RODRIGUES (1982) relatou que a taxa assimilatória líquida diminuía com a idade da planta. Estas divergências, talvez possam ser justificadas pela influência de condições climáticas, forma de condução dos experimentos e pelas variações intra específicas da TAL, sugeridas por WATSON (1952).

As diferenças nos valores de TAL, em funcão das variedades empregadas, também são referidas por OJIMA \& KAWASHIMA (1968); SANTOS FILHO et al. (1979), relatam que em duas linhagens de soja, a TAL atinge máximos valores aos 20 e 34 dias pós-emergência, respectivamente. Nas condições do experimento, obtiveram-se os valores máximos de TAL, para estilosantes, aos 28 dias pós-emergência, independente dos tratamentos utilizados, concordando com os relatos dos autores citados e respeitando as variabilidades entre as espécies.

Analisando-se o efeito dos diferentes níveis de cálcio nos valores da TAL, em cada intervalo, pode-se verificar que, de maneira geral, os maiores não foram aqueles obtidos no tratamento com maior nível de cálcio (T1), com exceção do terceiro intervalo. Os valores de TAL variam com 
TABELA 1. Taxa Assimilatória Líquida (TAL).

A - Resultados obtidos para Taxa Assimilatória Líquida (TAL), expressos em g/ $\mathrm{cm}^{2} \cdot$ dia. $10^{-3}$.

\begin{tabular}{ccccc}
\hline \hline \multirow{2}{*}{ Tratamentos } & \multicolumn{3}{c}{ Coletas } & \\
\cline { 2 - 5 } $\mathrm{T}_{1}$ & II - I & III - II & IV - III & V - IV \\
\hline & 0,553 & 0,365 & 0,132 & 0,088 \\
& 0,904 & 0,136 & 0,454 & 0,120 \\
& 0,757 & 0,302 & 0,285 & $-0,054$ \\
& & & & 0,169 \\
$\mathrm{~T}_{2}$ & 0,838 & 0,637 & 0,304 & 0,108 \\
& 1,025 & 0,188 & 0,508 & 0,242 \\
& 1,052 & 0,237 & 0,034 & 0,053 \\
& 1,371 & & & $-0,311$ \\
& 1,106 & 0,339 & $-0,048$ & 0,752 \\
$\mathrm{~T}_{3}$ & 1,173 & 0,277 & 0,309 & $-0,615$ \\
& & 0,409 & 0,140 & $-0,596$ \\
& 1,411 & & 0,695 \\
\hline \hline
\end{tabular}

B - Médias de tratamentos em cada coleta, de tratamentos e de coletas.

\begin{tabular}{cccccc}
\hline \hline & \multicolumn{5}{c}{ Colctas } \\
\cline { 2 - 6 } Tratamentos & II - I & III - II & IV - III & V - IV & Médias \\
\hline$n_{1}$ & 0,738 & 0,268 & 0,291 & 0,051 & 0,337 \\
$T_{2}$ & 0,972 & 0,354 & 0,282 & 0,173 & 0,445 \\
$T_{3}$ & 1,217 & 0,342 & 0,133 & 0,165 & 0,464 \\
$T_{4}$ & 1,091 & 0,187 & 0,237 & $-0,635$ & 0,220 \\
Médias & 1,004 & 0,288 & 0,236 & $-0,062$ & - \\
\hline \hline
\end{tabular}


TABELA 2. Taxa de Crescimento Relativo (TCR).

A - Resultados obtidos para Taxa de Crescimento Relativo (TCR), expressos em g/g.dia.10-1.

\begin{tabular}{|c|c|c|c|c|}
\hline \multirow{2}{*}{ Tratamentos } & \multicolumn{4}{|c|}{ Coletas } \\
\hline & II - I & III - II & IV - III & $V-I V$ \\
\hline \multirow{3}{*}{$T_{1}$} & 0,899 & 0,560 & 0,204 & 0,123 \\
\hline & 1,466 & 0,210 & 0,698 & 0,168 \\
\hline & 1,252 & 0,470 & 0,434 & $-0,071$ \\
\hline \multirow{3}{*}{$\mathrm{T}_{2}$} & 1,109 & 0,753 & 0,342 & 0,187 \\
\hline & 1,424 & 0,233 & 0,622 & 0,132 \\
\hline & 1,349 & 0,304 & 0,042 & 0,282 \\
\hline \multirow{3}{*}{$T_{3}$} & 1,667 & 0,353 & $-0,053$ & 0,060 \\
\hline & 1,414 & 0,328 & 0,366 & $-0,384$ \\
\hline & 1,538 & 0,464 & 0,140 & 0,638 \\
\hline \multirow{3}{*}{$T_{4}$} & 1,160 & 0,525 & 0,155 & $-0,767$ \\
\hline & 1,448 & 0,268 & 0,255 & $-0,491$ \\
\hline & 1,686 & $-0,083$ & 0,411 & $-0,678$ \\
\hline
\end{tabular}

B - MÉdias de tratamentos em cada coleta, de tratamentos e de coletas.

\begin{tabular}{cccccc}
\hline \hline & \multicolumn{5}{c}{ Coletas } \\
\cline { 2 - 6 } Tratamentos & II - I & III - II & IV - III & V - IV & Médias \\
\hline$T_{1}$ & 1,206 & 0,413 & 0,445 & 0,073 & 0,534 \\
$T_{2}$ & 1,294 & 0,430 & 0,335 & 0,201 & 0,565 \\
$T_{3}$ & 1,540 & 0,382 & 0,151 & 0,104 & 0,544 \\
$T_{4}$ & 1,431 & 0,237 & 0,274 & $-0,645$ & 0,324 \\
Médias & 1,368 & 0,365 & 0,301 & $-0,067$ & - \\
\hline \hline
\end{tabular}


TABELA 3. Razão Alfa.

A - Resultados obtidos para Razão Alfa, expressos $\mathrm{em} \mathrm{cm}^{2} \cdot \mathrm{g}^{-1}$.

\begin{tabular}{|c|c|c|c|c|}
\hline \multirow{2}{*}{ Tratamentos } & \multicolumn{4}{|c|}{ Coletas } \\
\hline & II - I & III - II & IV - III & V - IV \\
\hline \multirow{3}{*}{$T_{1}$} & 0,79 & 1,17 & 0,57 & 0,59 \\
\hline & 0,89 & 1,39 & 0,87 & 0,78 \\
\hline & 0,85 & 1,22 & 0,68 & 1,95 \\
\hline \multirow{3}{*}{$T_{2}$} & 0,92 & 0,88 & 1,06 & 0,76 \\
\hline & 0,96 & 0,46 & 1,18 & 0,10 \\
\hline & 1,02 & 0,89 & 0,07 & 0,89 \\
\hline \multirow{3}{*}{$\mathrm{T}_{3}$} & 0,84 & 1,14 & 0,48 & 0,95 \\
\hline & 0,98 & 0,70 & 1,28 & 1,11 \\
\hline & 0,76 & 1,37 & $-1,82$ & 1,29 \\
\hline \multirow{3}{*}{$\mathrm{T}_{4}$} & 0,88 & 0,81 & 1,57 & 1,04 \\
\hline & 1,03 & 0,78 & $-0,28$ & 1,15 \\
\hline & 1,06 & 0,75 & 0,50 & 1,06 \\
\hline
\end{tabular}

B - Médias de tratamentos em cada coleta, de tratamentos e de coletas.

\begin{tabular}{cccccc}
\hline \hline & \multicolumn{5}{c}{ Coletas } \\
\cline { 2 - 6 } Tratamentos & II - I & III - II & IV - III & V - IV & Médias \\
\hline$T_{1}$ & 0,84 & 1,26 & 0,71 & 1,11 & 0,98 \\
$T_{2}$ & 0,97 & 0,74 & 0,77 & 0,58 & 0,77 \\
$T_{3}$ & 0,86 & 1,07 & $-0,06$ & 1,12 & 0,75 \\
$T_{4}$ & 0,99 & 0,78 & 1,79 & 1,08 & 1,16 \\
Médias & 0,92 & 0,96 & 0,80 & 0,97 & - \\
\hline \hline
\end{tabular}




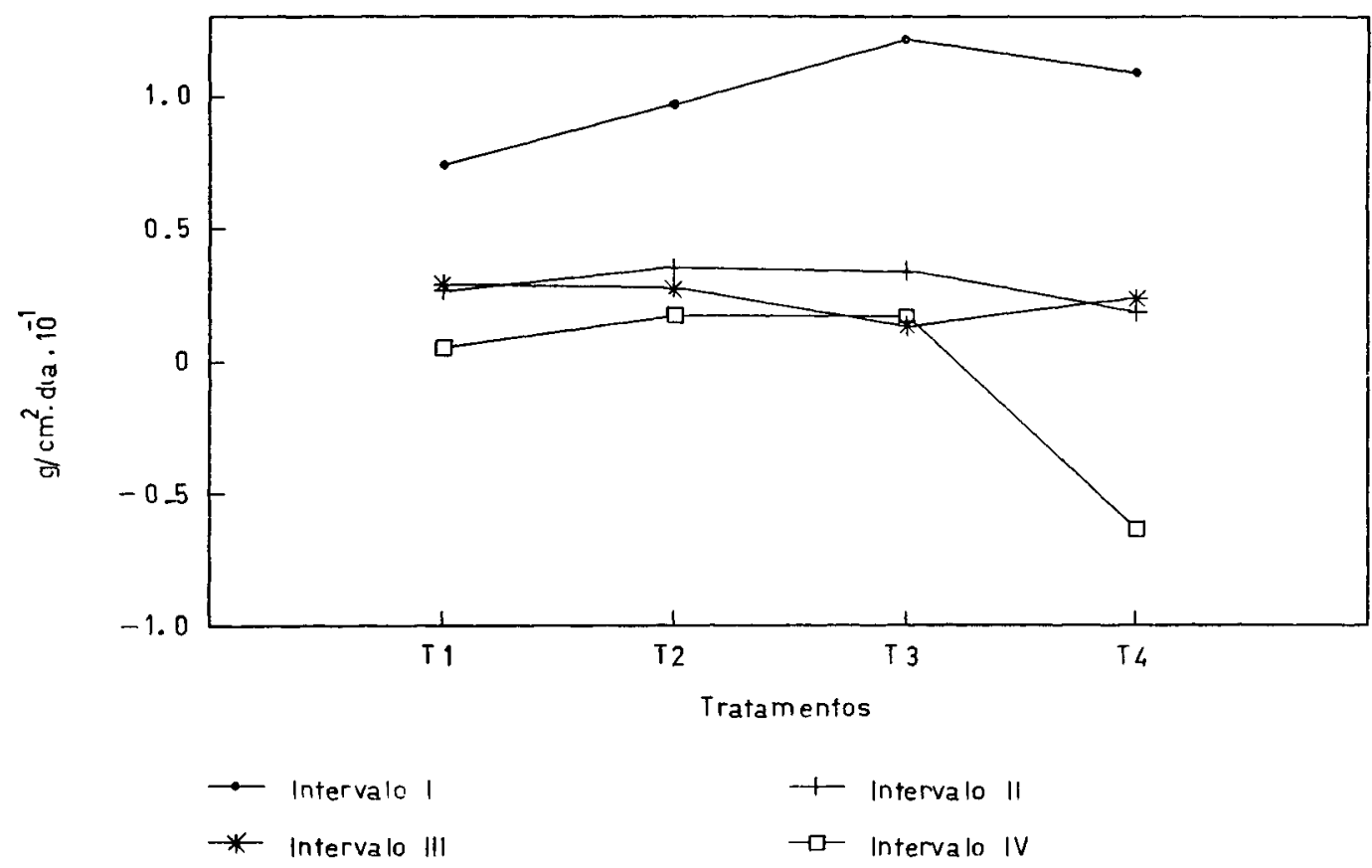

Figura 1 - Valores médios obtidos para Taxa Assimilatória Líquida $\left(\mathrm{em} \mathrm{g} / \mathrm{cm}^{2}\right.$. dia. $10^{-3}$ ), de plantas de estilosantes, submetidas a diferentes níveis de cálcio.

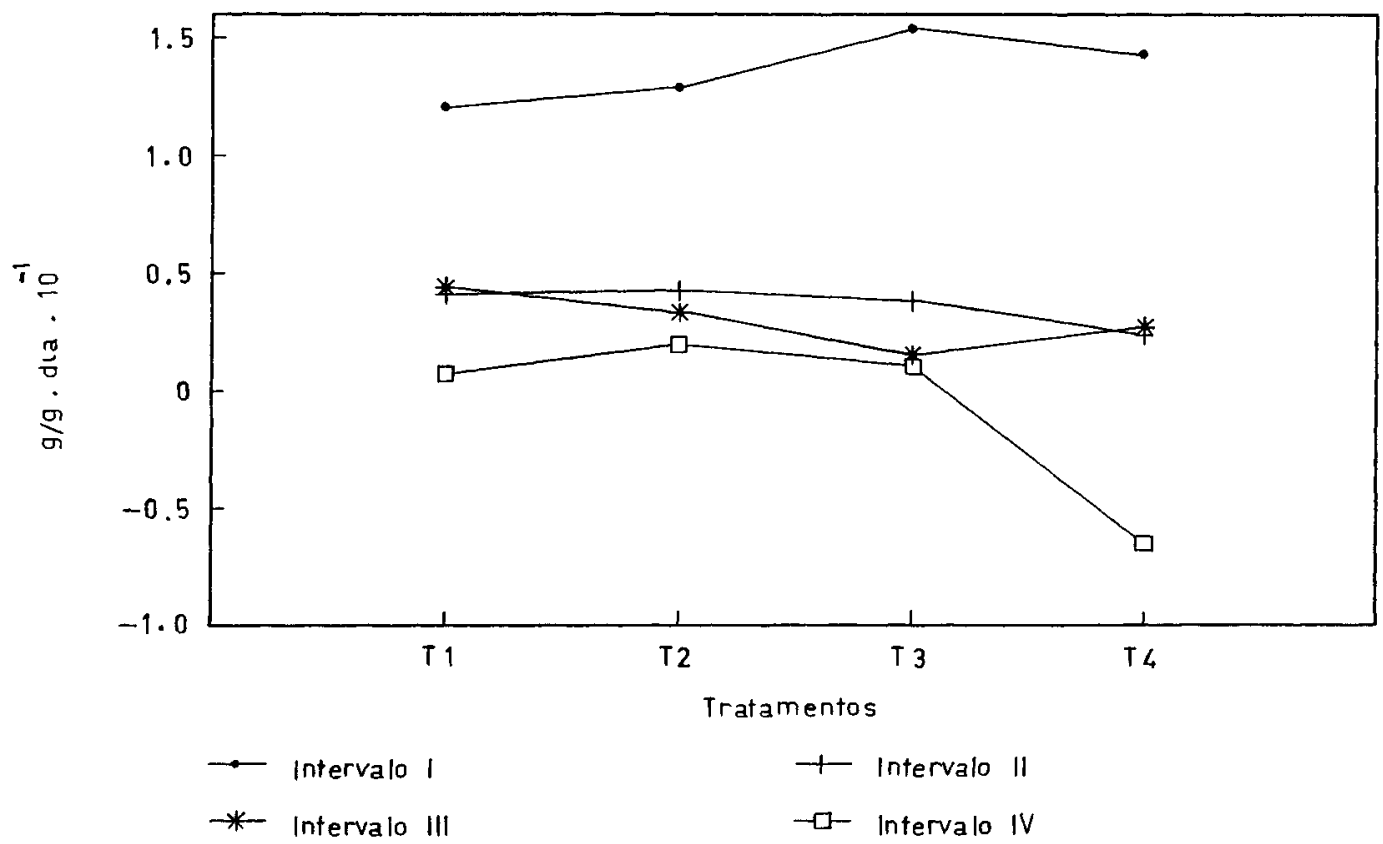

Figura 2 - Valores médios obtidos para Taxa de Crescimento Relativo (em g/g.dia.10-1), de plantas de estilosantes, submetidas a diferentes níveis de cálcio.

Sci. agric., Piracicaba, 50(1):45-57, fev./maio, 1993 


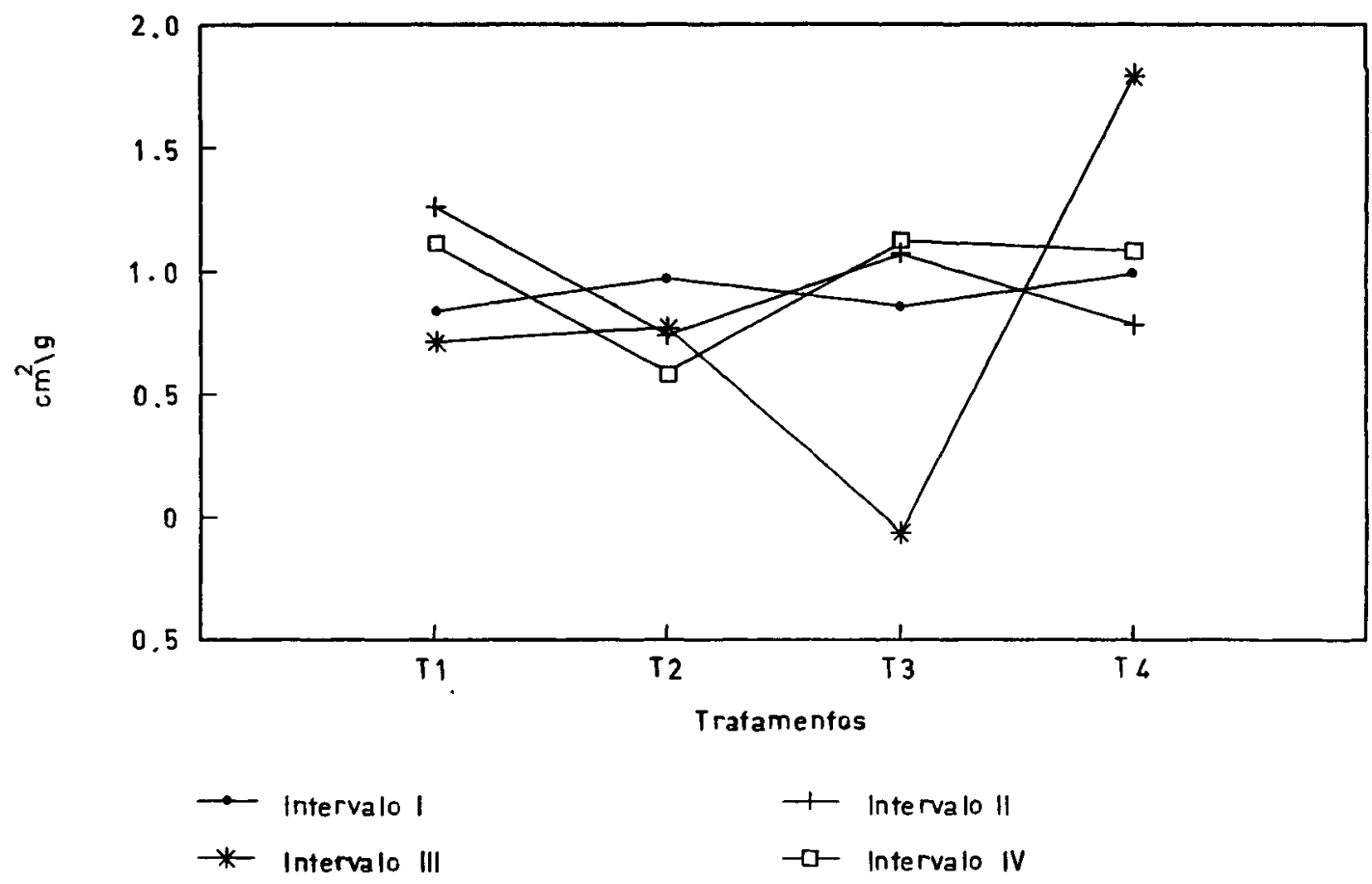

Figura 3 - Valores médios obtidos para Razão Alfa $\left(\mathrm{em}^{\mathrm{cm}^{2}} \cdot \mathrm{g}^{-1}\right)$, de plantas de estilosantes, submetidas a diferentes níveis de cálcio.

os níveis de cálcio, diferentemente, em cada intervalo. Assim, no primeiro, o tratamento T3, com baixo nível de cálcio $(66,66 \mathrm{mg}$ de cálcio/litro), foi o que apresentou maior TAL, vindo a seguir T4 (omisso em cálcio), enquanto que T1 (200 mg de cálciol litro) foi o responsável pelos menores valores de TAL. No segundo intervalo, T2 $(133,33 \mathrm{mg}$ de cálcio/litro) foi onde a TAL apresentou valores superiores aos demais tratamentos, sendo os menores aqueles do tratamento T4. O terceiro intervalo, caracterizou-se pela TAL ser maior no T1 e menor no T3. Já no quarto e último intervalo, no final da fase de desenvolvimento, os valores superiores de TAL aconteceram no tratamento $\mathrm{T} 2 \mathrm{e}$ os inferiores em T4; neste, ocorreu grande diminuição da área foliar. Assim, não fica clara a ação do cálcio na taxa assimilatória líquida de estilosantes, através do estudo do comportamento fisiológico das curvas componentes da Figura 1. Parece que, para cada idade estudada, ocorrem respostas diferentes para os níveis de cálcio, só apresentando pequenos valores de TAL no último intervalo, entre a quinta e a quarta coletas, quando o esperado, pela ação do cálcio na área foliar e matéria seca total, seria um efeito mais drástico na TAL em ausência de cálcio. Os trabalhos de WATSON (1952), de certa forma, dão alento a estes resultados, pois, segundo aquele autor, não há evidências da influência de níveis elevados de nutrientes sobre a TAL, ao mesmo tempo que sugere diminuição deste parâmetro, quando baixas concentrações de nutrientes são utilizadas.

Em 1986, BOARO relata que a TAL reflete a influência do sistema assimilador envolvido na produção de matéria seca, estimando a fotossíntese líquida; dessa forma, não se pode afirmar ser o cálcio indispensável para o sistema assimilador de plantas de estilosantes, a não ser em condições de ausência de cálcio, no final do período experimental, aos 80 dias da emergência. 


\section{Taxa de Crescimento Relativo}

A taxa de crescimento relativo (TCR) reflete o aumento da matéria orgânica seca, em gramas, de uma planta ou de qualquer órgão dessa, num intervalo de tempo, sendo função do tamanho inicial, ou seja, de material pré-existente (BENINCASA, 1988). Para BRIGGS et al. (1920), é a medida mais apropriada para avaliação do crescimento vegetal, sendo dependente de quantidade de material que está sendo acumulado. Seus valores constituem a TABELA 2 c Figura 2, pclas quais se observam, no primeiro intervalo, valores altos de TCR, com a diminuição desse parâmetro fisiológico nos intervalos subsequentes, tornando-se, inclusive, negativo no último intervalo, no tratamento omisso $\mathrm{cm}$ cálcio. Percebe-se, pois, uma primcira fase, compreendida pelo primeiro intervalo, de altos valores de TCR. A seguir, com queda acentuada, surge uma segunda fase, onde os valores de TCR do segundo e tereciro intervalos se agrupam, apresentando valores semelhantes, mas bem menores que aqueles encontrados no primeiro. Depois, o que se poderia denominar de fase terceira, onde TCR apresenta resultados menores ainda, inclusive com ponto de valor negativo, constitui o quarto $\mathrm{e}$ último intervalo.

Dessa forma, temos um período inicial de rápido acúmulo de material, seguido de uma fase de acúmulo mais ou menos constante, com um período final de declínio da TCR, cm função da queda ou morte de folhas; isto, provavelmente, ocorreu neste estudo, tendo $\mathrm{cm}$ vista seu encerramento ao final da fase vegetativa, condição agravada pela ausência de cálcio. No presente trabalho, a taxa de crescimento relativo foi negativa apenas em plantas submetidas à carência de cálcio, tratamento $T 4$, no quarto intervalo.

Os resultados aqui obtidos com plantas de estilosantes, referentes à TCR, concordam com os obtidos por vários autores, trabalhando com diferentes plantas. Já em 1920 , em trabalho que se tornou clássico, BRIGGS, et al. relataram o fato da TCR não ser constante durante o crescimento do vegetal, onde este apresentaria uma fase de desenvolvimento rápido da $T C R$, seguido de um declínio relativamente contínuo. Segundo os mesmos autores, a TCR é um parâmetro de extrema validade para comparação entre tratamentos. Em soja cv "Wayne", a TCR diminui com a idade, em função do autosombreamento e da atividade respiratória, cuja importância aumenta com a idade do vegctal (SIVAKUMAR \& SHAW, 1978). SANTOS FILHO et al. (1979), com duas linhagens da mesma espécie, relatam que a TCR decresceu continuamente, a partir de valores iniciais relativamente altos. Não se pode deixar de destacar aqui a contribuição de MILTHORPE \& MOOREY (1974), os quais, em extenso trabalho de revisão da análise de crecimento de plantas, submetidas às condições usuais, relataram que durante a ontogenia de uma cultura, há um primeiro período com taxa de crescimento acclerada, seguido de um outro em que as taxas são mais ou menos constantes e de um terceiro, com declínio neste parâmetro. Neste último período, o crescimento se torna negativo em função da morte de folhas e gemas. Em 1982, RODRIGUES, com plantas de soja, e BOARO (1986), em feijão, obtiveram resultados semelhantes quanto ao comportamento da TCR, aos aqui obtidos para estilosantes, os quais, por sua vez, estão de acordo com os referidos pelos inúmeros autores citados.

A influência dos diferentes tratamentos de cálcio utilizados, dentro de cada intervalo, nos valores da taxa de crescimento relativo, pode ser observada pela Figura 2. No primeiro intervalo, há tendência de aumento da TCR com a diminuição dos níveis de cálcio, ocorrendo o máximo valor no tratamento T3, vindo, a seguir, o tratamento ausente em cálcio (T4). O comportamento da TCR, no segundo intervalo, mostra resultados semelhantes para os tratamentos T1 (200 mg de cálcio/litro), T2 $(133,33 \mathrm{mg}$ de cálcio/litro) e T3 $(66,66 \mathrm{mg}$ de cálcio/litro), com leve aumento $\mathrm{em}$ T2 e pequeno declínio $\mathrm{cm}$ T4 (scm cálcio). No terceiro intervalo, os valores de TCR caem com a queda dos níveis de cálcio, até o tratamento T3, que apresenta menor resultado, com ascensão da TCR no tratamento T4. O quarto e último intervalo apresenta o maior valor de TCR no tratamento T2, seguido pelo T3 e depois T1. Neste intervalo, o tratamento omisso em cálcio (T4) apresentou resultados de crescimento negativo, evidenciando nesta fase da vida da planta de estilosantes, em função do final do ciclo vegetativo, uma forte influência da ausência do mineral, que pode, possivelmente, entre outros processos fisiológicos, ter acelerado a abscisão das folhas, como a literatura é unânime em reconhecer (COBRA NETO ct al., 1971; MALAVOLTA et al., 1976).

Estes resultados obtidos para TCR, em cada intervalo, nos diferentes níveis de cálcio utilizados e nas condicões citadas, mostram respostas relacionadas à idade das plantas de estilosantes. Assim, a variabilidade dos resultados 
não permite definir um padrão de comportamento da influência do cálcio, na taxa de crescimento relativo, a não ser, possivelmente, recomendar a utilização de cálcio na fase de crescimento mais avançada, tentando evitar crescimento negativo. RODRIGUES (1982), trabalhando com soja em meio nutritivo completo e omisso em cálcio, obteve resultados semelhantes, com grande variação nos resultados de TCR em função do intervalo considerado, notando, no entanto, que ao final do ciclo de desenvolvimento, as plantas carentes em cálcio apresentavam, no mesmo intervalo, valores de TCR menores do que aquelas cultivadas em solução completa. Apesar de saber-se que a concentração do cálcio varia de espécie para espécie, condições do meio, idade da planta (CAROLUS, 1975), o que possivelmente explicam as variações na TCR dentro de cada intervalo considerado, os resultados de RODRIGUES (1982), apesar de obtidos para soja, respaldam os aqui auferidos para estilosantes, relativamente à taxa de crescimento relativo.

\section{Razāo alfa}

Os dados obtidos relativos à razão alfa constituem a TABELA 3 e Figura 3. A observação dessa figura, mostra que, entre os intervalos de coleta, ocorrem variações entre os valores de razão alfa, de tal maneira que os maiores são aqueles encontrados no quarto e último intervalo, enquanto os menores estão no terceiro intervalo de coleta. Há um aumento deste parâmetro do primeiro para o segundo intervalo, queda no terceiro e ascensão no último, isto sem levar em conta a influência dos vários tratamentos de cálcio, mas apenas o próprio desenvolvimento das plantas de estilosantes.

Considerando-se o efeito dos diferentes níveis de cálcio empregados, ao longo do experimento, pode-se notar os maiores valores da razão alfa no tratamento omisso em cálcio (T4), enquanto os menores estão no tratamento $T 3$, seguido do $\mathrm{T} 2$, apresentando o tratamento usual (T1), valores próximos da unidade, abaixo apenas do T4.

Analisando-se, através da Figura 3, o efeito dos vários níveis de cálcio na razão alfa, dentro da mesma idade fisiologica da planta, representado pelo cálculo de cada intervalo, verifica-se que as concentraçöes de cálcio respondem diferentemente, dependendo do nível desse elemento e do intervalo considerado. Assim, no primeiro intervalo, com cerca de 38 dias pós- emergência do estilosantes, nota-se uma tendência de aumento da razão alfa, do tratamento usualmente empregado na solução $n^{\circ} 1$ de HOAGLAND \& ARNON (1950), ou seja, do T1 ao T4, ausente em cálcio, com picos em T2 e queda em T3. No segundo intervalo, os maiores valores encontram-se no $T 1$, os menores em $T 2$, enquanto $T 3$ apresenta - segundo valor e T4 pouco acima de T2. 0 terceiro intervalo caracteriza-se por ser aquele onde surge o único resultado negativo obtido neste experimento para razão alfa, que ocorre no tratamento T3. O tratamento T4 apresenta um extraordinário pico, com os maiores valores do intervalo e também de todo o período experimental, enquanto $\mathrm{T} 1 \mathrm{e}$ T2 mostraram valores intermediários, sendo T2 maior que T1. No quarto intervalo, os níveis de cálcio dos tratamentos $\mathrm{T} 3$ e $\mathrm{T} 1$, pela ordem, são aqueles de maiores valores de razão alfa, enquanto o menor valor ocorre no tratamento T2, ficando T4 próximo aos dois maiores.

Em função dos resultados obtidos, parece difícil definir o comportamento de plantas de estilosantes, relativamente à razão alfa, no tocante a influência dos vários níveis de cálcio nesse parâmetro fisiológico. A variabilidade dos resultados obtidos em função da idade da planta, apesar de ser destacado por trabalhos como os de CAROLUS (1975), não satisfaz ao não definir uma tendência dos valores de razão alfa de estilosantes, em função dos diferentes tratamentos de cálcio empregados. Inclusive, deve se destacar a enorme oscilação ocorrida no terceiro intervalo, entre os tratamentos T3, com baixo nível de cálcio $(66,66$ $\mathrm{mg}$ de cálcio/litro) e valor negativo para a razão alfa e o T4, carente em cálcio com os maiores valores para este parâmetro, obtido em todo o periodo experimental. Apesar disso, ou melhor, talvez em função disso, ao longo do trabalho, os resultados mais expressivos de razão alfa aconteceram no tratamento $\mathrm{T} 4 \mathrm{e}$, em seguida, no T1, com concentração de cálcio que seria usualmente utilizado na condução de qualquer trabalho em cultivo hidropônico.

No entanto, o próprio CAROLUS (1975) afirma que as diferentes especies, ou mesmo variedades, respondem diferentemente aos níveis de cálcio empregados. Logo, resultados obtidos para outras plantas podem não concordar com este, sem invalidar os aqui obtidos. RODRIGUES (1982) encontrou, em soja, valores de razão alfa que aumentaram do primeiro para o segundo intervalo, no tratamento completo, decrescendo posteriormente. Para o tratamento omisso, a mesma 
autora relata aumentos neste parâmetro até o terceiro intervalo, caracterizado por um pico gráfico, seguido de queda no quarto intervalo. Esse pico, obtido no terceiro intervalo, foi aquele onde surgiram os maiores valores de razão alfa, no trabalho em questão. Dessa maneira, os resultados assemelham-se aos conseguidos pela autora supra citada, considerando-seapenas tratamentos completo e ausente em cálcio. Para tratamentos de níveis intermediários e seus efeitos na razão alfa, a literatura é extremamente pobre.

Apesar de não ficar bem definida a ação do cálcio neste parâmetro, parece válida a discussão dessa relação alométrica e sua relação com os processos fisilógicos que ocorrem em uma planta. Sabe-se que o crescimento vegetal está fortemente relacionado ao meio e, Whitehead \& Myerscouch (1962), citados por EVANS (1972), discutem o crescimento sob a forma de "entidades fotossintéticas", introduzindo o conceito de superávit de produção de matéria seca, utilizadas para manter a planta, fornecendo condiçōes para a fase reprodutiva ou mesmo de rebrota vegetativa. Este superávit poderia ser definido como relação logarítmica da área foliar e da matéria seca total da planta, relação essa que, em 1974, MILTHORPE \& MOOREY chamaram de razão alfa, definindo ainda que se alfa fosse igual a 1 , a taxa de crescimento dos parâmetros envolvidos seria igual, enquanto que se fosse diferente da unidade, significaria mudanças na forma e na função. HUNT (1978) diz que a razão alfa serviria como índice de tamanho, indicando participação de assimilados, sendo fortemente influenciada pelo meio e, especialmente, pela nutrição mineral, mormente estresse nutricional. Em 1979, MAGALHÃES atenta para o fato de que a razão alfa, ao descrever uma relação alométrica entre área foliar e matéria seca, mostra como a proporção relativa dos tecidos assimilados se alteraria durante o crescimento de uma planta, o que, para RODRIGUES (1982), indica a direção dos assimilados.

Logo, a discussão por este prisma mostra grandes alterações na movimentação de assimilados nas plantas de estilosantes, em função da idade e também dos níveis de cálcio possivelmente com a associação de ambos e de outros fatores. A omissão do cálcio, mostrou ser extremamente mais efetiva na redução da matéria seca total do que na redução da área foliar, principalmente no terceiro e, em menor escala, no quarto intervalo, dados concordantes com
HELMS \& MYERS (1972), OLIVEIRA et al., (1975), PEEDIN \& MacCANTES (1977), MALAVOLTA (1980) e, principalmente, RODRIGUES (1982). No entanto, outros autores como LONERAGAN (1959), COBRA NETO et al., (1971), MALAVOLTA et al., (1976), GALLAHER et al., (1976) e ROSOLEM (1980), em soja e outras espécies, em meio carente em cálcio, relatam a efetiva redução de área foliar. A análise do tratamento completo $\mathrm{T} 1$ revela valores de razão alfa, demonstrando a ocorrência de desvio de assimilados para a área foliar, no início do desenvolvimento vegetal, entre o primeiro e segundo intervalo, com aumento novamente no quarto intervalo, final da fase vegetativa, de certa forma reforçando a idéia de Whitehead \& Myerscouch (1962), citados por EVANS (1972), do superávit necessário para o início da fase reprodutiva.

Então, a partir dos resultados de razão alfa pode-se, possivelmente, inferir um maior desenvolvimento da área foliar ou maior acúmulo de matéria seca em raízes, ramos, etc em funçào dos vários níveis de cálcio empregados, relacionando-se com a espécie vegetal, a idade das plantas e fatores do meio, como temperatura, luminosidade, umidade relativa, etc. Não se devem utilizar isoladamente os dados de razão alfa sem correlacioná-los com os ítens levantados acima, sob pena de incorrer-se em erros de avaliação. Isso deve-se à movimentação de assimilados, desenvolvimento da área foliar, acúmulo de matéria seca e ao crescimento de uma planta, dados que estão alométricamente relacionados como razão alfa, que são dependentes em maior escala do nível nutricional e da idade do vegetal. Estes alteram fundamentalmente a fisiologia de uma planta sendo, portanto, indispensáveis à análise global, para a interpretação dos resultados de razão alfa.

\section{CONCLUSÕES}

Apesar de algumas variações terem sido observadas pode-se concluir que, nas condições estudadas, plantas de Stylosanthes guyanensis (Aubl.) Sw. cv. Cook, durante a fase de crescimento vegetativo, alcançaram melhor desenvolvimento traduzido $\mathrm{em}$ maiores valores de TAL e TCR, em níveis de 200 e $133,33 \mathrm{mg}$ de cálcio/litro de solução nutritiva, mostrando-se exigente ao fornecimento desse mineral. 


\section{REFERÊNCIAS BIBLIOGRÁFICAS}

ANDREW. C.S. Influence of nutrition on nitrogen fixation and growth of legs. In: HUNLAY, B. A review of nitrogen and tropics with particular references pastures. Comm. Bur. Past. Fed. Crop. Bulletin, London, v.46, p.130-146, 1962.

ASCÊNCIO, J.; FARGAS, J. E. Análisis del crescimiento del frijol (Phaseolus vulgaris L. var. "Turrialba-4") cultivado en la solucion nutritiva. Turrialba, San Jose, v.23, p.420-428, 1973.

BANZATTO, D.A.; KRONKA, S.N. Experimentaçāo agricola. Jaboticabal, FUNEP : 1989. 247p.

BARRIGA, J.P. Autoecologia de Stylosanthes humilis HBS: avaliação da viabilidade morfológica e estudos da biologia da semente. Piracicaba, 1979. 97p. Dissertação - (Mestrado) - Escola Superior de Agricultura "Luiz de Queiroz", Universidade de São Paulo.

BENINCASA, M.M.P. Análise de crescimento de plantas; noçōes básicas. Jaboticabal : FUNEP, 1988. 42p.

BOARO, C.S.F. Influência da variação dos níveis de magnésio sobre o desenvolvimento do feijoeiro (Phaseolus vulgaris L. cv. Carioca), em cultivo hidropônico. Botucatu, 1986. Dissertação - (Mestrado) - Faculdade de Ciências Agronômicas de Botucatu, Universidade Estadual Paulista "Julio de Mesquita Filho".

BRIGGS, G.E.; KIDD, M.A.; WEST, A.R.C.S. A quantitative analysis of plant growth. Annales of Applied Biology, Cambridge, v.7, p.202-223, 1920.

BUTTERY, B.R.; BUZZELL, R.I. Some differences between soybean cultivars observed by growth analysis. Canadian Journal of Plant Science, Ottawa, v.52, p.13-20, 1972.

CAROLUS, R. Calcium related physiological disorders. Communications in Soil Science and Plant Analysis, New York, v.6, p.294-299, 1975.

COBRA NETO, A,; ACCORSI, W.R., MALAVOLTA, E. Estudos sobre a nutrição do feijoeiro (Phaseolus vulgaris L. var. Roxinho). Anais da Escola Superior de Agricultura "Luiz de Queiroz", Piracicaba, v.28, p.257-273, 1971 .

EIRA, P.A. et al. Fatores nutricionais limitantes do desenvolvimento de três leguminosas forrageiras em solo Podź́lico vermelho-amarelo. Rio de Janeiro, V Reuniāo Latino Americana de Rhyzobium, 1970. 23p.
EVANS, G.C. The quantitative analysis of plant growth. Berkeley : University of California, 1972. $734 p$.

FRANÇA, G.E.; CARVALHO, M.M. Ensaio exploratório de fertilizantes de cinco leguminosas tropicais em solo de cerrado. Pesquisa Agropecuária Brasileira, Brasilia, v.5, p.140-153, 1970.

GALLAHER, R.N.; BROWN, R.H.; ASHLEY, D.A.; JONES JR., J.B. Photosynthesis and ${ }^{14} \mathrm{CO}_{2}$ photosynthate translocations from calcium-deficient leaves of crops. Crop Science, Madison, v.16, p.116119,1976

HELMS, E.; MYERS, N. Responses of bean sprouts (Phaseolus vulgaris $\mathrm{L}$. and Vigna sinensis End.) to calcium. Thai Journal Agricultural Science, Bang Kok, v.5, p.15-21, 1972.

HOAGLAND, D.R.; ARNON, D.I. The water method for growing plants without soil. Berkeley : University of California, College of Agriculture, 1950. 32p. (Circular, 343).

HUNT, R. Plant growth analysis. London : E. Arnold, 1978. $67 \mathrm{p}$.

KUMURA, A.; NANIWA, I. Studies on dry matter production of soybean plants. I. Ontogenic changes in photosynthesis and respiratory capacity of soybean plant and its parts. Proceedings of Crop Science Society of Japan, Tokyo, v.33, p.467-472, 1965.

LONERAGAN, J.F. Calcium in the nitrogen metabolism of subterranean clover. Australian Journal of Biological Sciences, East Melbourne, v.12, p.26-39, 1959.

MALAVOLTA, E. Elementos de nutriçăo mineral de plantas. São Paulo : Ceres, 1980. 251p.

MALAVOLTA, E.; CHAVES, I.; TONIN, G.S.; SOUZA, A.F. Deficiências de macronutrientes na soja (Glycine max L. Merril, var. IAC-2). Anais da Escola Superior de Agricultura "Luiz de Queiroz", Piracicaba, v.33, p.471-477, 1976.

MAGALHĀES, A.C.N. Análise quantitativa de crescimento. In: FERRI, M.G. (Coord.). Fisiologia vegetal. São Paulo : EPU/EDUSP, 1979. p.331-350.

MILTHORPE, F.L.; MOOREY, J. An introduction to crop physiology. Cambridge : Cambridge University Press, 1974. 201p.

NORRIS, D.O. The role of calcium and magnesium in the nutrition of Rhyzobium. Australian Journal of 
Agricultural Research, East Melbourne, v.10, p.651$698,1959$.

NORMAN, A.G. Soybean; physiology, agronomy and utilization. New York : Academic. 1975. 249p.

OJIMA, M.; KAWASHIMA, R. Studies on the seed production of soybean. 5. Varietal differences in photosynthetic rate of soybean. Proceedings of Crop Science Society of Japan, Tokyo, v.37, p.667-678, 1968.

OLIVEIRA, G.D.; HAAG, H.P.; DECHEN, A.R.; FERNANDES, P.D. Nutrição mineral de hortaliças. Carência de micronutrientes em beringela (Solanum melongena L.). Anais da Escola Superior de Agricultura "Luiz de Queiroz", Piracicaba, v.32, p.671-680, 1975 .

PEEDIN, G.F.; MacCANTES, C.B. Influence of variety and soil application of $\mathrm{Ca}$ on development of $\mathrm{Ca}$ defficiency in tobbaco. Agronomy Journal, Madison, v.69, p. 7 I-77, 1977.

RODRIGUES, J.D. Influência de diferentes niveis de cálcio, sobre o desenvolvimento de plantas de estilosantes (Stylosanthes gityanensis (Aubl.) Sw. cv. Cook), em cultıvo hidropônico. Botucatu, 1990. p. Tese (Livre-Docência) - Faculdade de Ciências Agronômicas de Botucatu, Universidadi Estadual Paulista "Julio de Mesquita Filho".

RODRIGUES, S.D. Análise de crescimento de plantas de soja (Glycine mar (L.) Merril) submetidas à carências nutricionais. Rio Claro, $1982 . \quad$ p Dissertação (Mestrado) - Instituto de Biociencias, Universidade Estadual Paulista "Julio de Mesquita Fılho".

ROSOLEM, C.A Nutrição mineral e adubação da soja. Piracicaba, Instltuto da Potassa, $1980 . \quad 80 \mathrm{p}$. (POTAFOS. Boletim TÉcnico, 6).
SANTOS FILHO, B.G. et. al. Análise de crescimento de duas linhagens de soja (Glycine max L. Merril), em Pelotas. In: SEMINÁRIO DE PESQUISA DE SOJA, 1., 1979, Londrina. Anais... Londrina, v.3, p.348$361,1979$.

SESTAK, Z.; CATSKY, I.; JARVIS, P.G. Assessment of least area and other assimilating surfaces. In:_. Plant phytosynthetic production. The Hague: W. Junk, 1971. cap. 14, p.517-555.

SIVAKUMAR, M.V.K.; SHAW, R. H. Methods of growth analysis in field grown soybean (Glycine max (L.) Merril). Annals of Botany, London, v.42, p.213222, 1978.

VASCONCELOS, C.N.; ASSIS, A.G.; SOUZA, R. M.; VILASSA, H.A.; GARCIA, R.; CHRISTMAS, E. P. Estudo do valor nutritivo $e$ produção de cinco leguminosas tropicais na zona da mata de Minas Gerais. Revista da Sociedade Brasileira de Zootecnia, Viçosa, v.3, p.30-33, 1974.

WATSON, D.J. The physiological basis of variation in yield. Advances in Agronomy, New York, v.4, p.101-145, 1952.

WHYTE, R.O.; NILSSON-LEISSNER, G.; TRAUBLE. H.C. Las leguminosas en la agricultura. Roma, FAO, 1968. 405p. (Estudios Agripecuários, 21).

Trabalho entregue para publicação em 06.05 .92 Trabalho aprovado para publicação em 24.06.92 